\title{
A propos du genou douloureux de la jeune fille Peut-on éviter les pièges psychologiques de la névrose ?*
}

\author{
About knee pain in young woman. Are neurotic pittfalls avoidable?
}

\author{
M. Patris
}

Service de Psychiatrie Adultes, Clinique Psychiatrique, Hôpitaux Universitaires de Strasbourg, BP 426, F-67091 Strasbourg Cedex

Je me suis permis de modifier le titre de ma communication, plutôt que de parler de "Rotule et pôle de fixation", j'ai proposé comme titre "A propos du genou douloureux de la jeune fille" et en sous-titre "Peut-on éviter les pièges psychologiques de la névrose?".

Autrement dit, mon propos se situe au niveau de la prévention de la iatrogénèse. Ma mission ici est en fait très délicate, car le dialogue entre chirurgien et psychiatre n'est pas toujours facile à engager et, pour trouver un terrain d'entente, il est souvent nécessaire que s'instaurent au préalable des liens de confiance voire des liens d'amitié entre collègues appartenant à chacune des disciplines.

Avant toute formation psychiatrique, j'ai été confronté, parfois de manière dramatique, aux liens étroits qui existent entre le terrain psychologique des patients et leur pathologie orthopédique. En d'autres termes, cela peut paraître paradoxal, c'est dans un service d'orthopédie que s'est éveillé mon intérêt pour l'hystérie, la psychosomatique et 1'hypochondrie, trilogie que j'ai reprise dans le titre de ma thèse.

Ainsi ma modeste expérience de la

* Conférence faite à la XVIIe Réunion du GECO, Les Arcs (16 au 21 janvier 1993)

Code Méary : 0404.0 chirurgie orthopédique m'a convaincu à quel point il était nécessaire de s'assurer des services d'un psychothérapeute compétent pour éviter tant que faire se peut certains pièges psychologiques.

Je ne pense donc pas que les rapports entre chirurgiens et psychiatres soient fatalement conflictuels. Certes, les premiers ont tendance à considérer les psychiatres comme des rêveurs, des poètes de la médecine enfermés dans un langage hermétique, certains prétendent même que la fréquentation des malades mentaux n'est pas sans conséquence sur leur propre équilibre. Les psychiatres quant à eux sont parfois tentés de reprocher aux chirurgiens de penser avec leur scalpel, ou de commencer à penser après l'intervention, voire même de ne jamais penser. Bien qu'excessives, ces caricatures ne sont pas à mon sens inutiles. Chacun a besoin de se rassurer en marquant bien ses différences et à partir de là, on peut commencer à discuter.

Chacun devrait donc s'en tenir à son rôle et savoir recourir à l'autre à bon escient. J'hésiterais quant à moi à confier mes os à un orthopédiste qui interpréterait mes rêves avant de prendre une décision opératoire.

Cela nous amène sur le terrain de la psychologie médicale ou si on préfère de la psychosomatique qui partent toutes deux de l'hypothèse de l'incidence du psychisme sur l'éclosion, l'évolution et la guérison d'une pathologie indiscutablement organique dans certains cas, soit aussi de syndromes purement fonctionnels quand il s'agit de pathologie névrotique.

Puisqu'il est question aujourd'hui de rotules et par conséquent de genoux, notamment des jeunes filles, se pose la question d'une spécificité de cette atteinte quant au terrain psychologique. Les chirurgiens orthopédistes semblent avoir déjà répondu en relevant la fréquence de la coïncidence d'un certain "terrain psychologique" et les problèmes de genou. Il s'agirait souvent d'adolescentes chez qui le symptôme orthopédique serait fréquent sous une forme bénigne, voire tout à fait physiologique, et qui pourraient occasionnellement utiliser ce symptôme banal pour exprimer un malaise névrotique. En d'autres termes, à partir d'une rotule douloureuse, certaines adolescentes font une véritable fixation, ce qui est un comble pour un bout d'os qui a une tendance naturelle à bouger dans tous les sens. Elles en feraient aussi un véritable drame, parfois avec la complicité de parents qui cherchent à médicaliser chez leur fille un problème au départ relationnel. Il peut s'agir, je le précise, d'un problème relationnel entre les parents eux-mêmes. Car c'est bien connu, le fait d'avoir un enfant malade peut constituer un abcès de fixation (voyez comme ce terme insiste) pour un couple sur le point de se désunir. Un 
enfant ou un adolescent peut se prêter totalement à son insu à ce jeu dont il fait évidemment les frais.

L'interprétation ici est facile : le signifiant genou peut facilement s'entendre comme l'acte de nouer. "Je noue, ce qui entre mes parents est en voie de se dénouer". Françoise Dolto avait fait une autre interprétation en juxtaposant les deux pronoms personnels, je comme je suis et nous comme nous sommes, dans une communication que j'ai exhumée pour me donner quelques idées. Ce jeu de mots : je - nous, peut paraître à première vue un jeu de mots facile mais il n'a pas d'autre ambition que d'illustrer sur quoi se joue le choix de tel ou tel symptôme hystérique. Car un tel symptôme exprime par le corps ce qui ne peut se dire en mots, ce qui au sens littéral est refoulé.

Voici l'histoire d'une adolescente que j'ai suivie il y a quelques années : cette charmante jeune fille, au demeurant anorexique m'avait été adressée après avoir été opérée des deux genoux pour ablation des ménisques. Les chirurgiens étaient frappés par sa passivité pour ne pas dire sa complaisance à se faire opérer. Ils se sentaient un peu culpabilisés car, a posteriori, leurs interventions auraient pu, de leurs propres aveux, être évitées. En fait, en prolongeant son séjour dans les Hôpitaux, cette jeune fille permettait à sa mère de rencontrer quotidiennement, en tout bien tout honneur, l'éminent Professeur, dont elle était l'amie très intime. Le symptôme de Blanche, appelons-la comme ça, l'avait aussi rapprochée indirectement de son père qu'elle adorait, mais comme malheureusement les lois en vigueur n'autorisent pas les jeunes filles à épouser leur papa, tout son roman oedipien était escamoté derrière un discours idyllique sur la prétendue bonne entente des parents. Elle déplaçait en d'autres termes l'amour qu'elle portait à son père sur le couple parental et ceci envers et contre toute évidence.

De telles constructions risquent, $\mathrm{j}$ 'en suis conscient, de vous renforcer dans l'idée que tous les psychiatres ont l'esprit tordu et a fortiori les psychanalystes. Néanmoins, ce que j'essaye de remonter pièce par pièce n'est ni une plaisanterie, ni une fable. Ceci au contraire vous met sous les yeux les éléments constitutifs du symptôme hystérique :

1. une totale complaisance à se faire opérer avec la bénédiction de toute la famille et de la faculté ;

2. un total déni de tout problème psychologique avec, au contraire, un discours parfaitement banalisant, lisse, rassurant, excluant tout problème relationnel et affectif ;

3. 1e refoulement de penchants affectifs, sinon sexuels, qui tombent sous le coup d'une censure implacable ; la puberté venant renforcer le refoulement des sentiments oedipiens.

L'inconscient, je ne vous apprends rien, et ceci n'est pas à prendre comme un reproche, n'est pas le domaine de prédilection de la chirurgie. Les chirurgiens sont par vocation pragmatiques, positifs et parfois plutôt interventionnistes. Un certain bon sens constitue à la fois les bases et l'essentiel de leurs compétences psychologiques. Ce sont pourtant eux, et ceci prenez-le comme un compliment, les premiers à pressentir intuitivement que certaines demandes, tout particulièrement celles venant d'adolescentes, de femmes séduisantes ou qui souhaiteraient le rester... répondent à une logique qui dépasse ce simple bon sens médical. Un acte chirurgical se charge toujours, même si ce n'est qu'à un très faible degré, d'une signification sexuelle. L'ignorer n'est pas la conséquence d'un manque de culture psychanalytique, mais celle du refoulement même car les chirurgiens ont aussi un inconscient, c'est ce qui, entre autre, leur permet d'avancer, imperturbables, tels de majestueux brise-glace à travers les banquises de l'hystérie.

La signification sexuelle de certains actes ne s'exprime que sous couvert d'humour, ainsi Henri Coudane ce matin, en nous montrant quelques membranes d'un genou béant, parlait, je cite "d'un véritable hymen qu'il faut ouvrir pour passer dans le cul-de-sac quadricipital de ces jeunes filles". Ouvrir un genou et a fortiori ouvrir les genoux peut exprimer par métaphore, un forçage sexuel, une défloration. L'adolescen- te dont je vous parlais tout à l'heure, Blanche, m'avait mis sur cette voie, en paroles je tiens à le souligner, au cours de nos entretiens psychothérapiques. Elle s'était fait ouvrir les genoux par l'amant de sa mère, une façon bien détournée de le lui "piquer", mais la seule que lui autorisait son sur-moi. Elle m'en donnait la clé en m'avouant qu'elle adorait la légende de Tristan et Iseut. Or dans cette histoire, Iseut promise à un roi qu'elle n'aime pas, ou qu'elle n'est pas censée aimer au regard d'une censure oedipienne puisque ce roi a l'âge de son père, fait le serment "qu'elle n'ouvrira jamais les genoux à un autre homme" que celui qui pourra répondre à certaines exigences. C'était du moins la version que Blanche m'avait relatée. C'est l'occasion pour moi de rappeler que les genoux d'une femme ont un intérêt érotique tout particulier. C'est non seulement ce qu'on regarde, faute de mieux, mais c'est aussi ce qu'on caresse pour en somme "tâter le terrain". A partir de là, la voie est ouverte et tous les espoirs sont permis. J'irai même plus loin, c'est une sorte de camp de base à partir duquel on peut rêver aux plus folles escalades.

Tout ceci est bien joli, me direzvous, mais quel intérêt peut avoir une pure et innocente créature, une adolescente, à s'offrir au scalpel, en l'occurrence très phallique, du chirurgien? Ma réponse risque de vous décevoir, le contenu de l'inconscient n'est jamais ni pur, ni innocent et les désirs qui sont maintenus en exil ne trouvent de satisfactions que sous des représentations déplacées, déformées, suffisamment méconnaissables, pour que le conscient les accepte.

Ce que j'ai évoqué à propos de Blanche, n'épuise pas toutes les significations symboliques du genou, loin de là. J'ai insisté sur l'aspect érotique, non par provocation, mais parce que l'adolescen$c e$, articulation psychologique entre l'enfance et l'âge adulte, plonge chaque sujet dans une forme de désarroi, voire d'angoisse, devant l'éveil de ses propres pulsions sexuelles et tout le drame oedipien, en sommeil depuis des années, se réveille avec une force terrifiante : la métamorphose du corps en rend théori- 
quement possible la réalisation.

Ces considérations nous éloignent dangereusement d'une orthopédie mécaniste, physique, avec ses forces, ses pressions, ses angles, notamment l'angle " $Q$ " que j'ai découvert ce matin. Les psychiatres aussi rêvent parfois d'une psychologie construite solidement, rationnellement sur les bases de la logique formelle ou de la linguistique structurale. Ce rêve est sans espoir. Notre discipline puise son sérieux ailleurs que dans les sciences exactes; elle s'appuie sur des lignes de force occultes, codées dans ce qui se dit entre patient et thérapeute.

Je voudrais passer à un autre cas clinique, qui rentre tout à fait dans la perspective de la prévention de la iatrogénèse. Pour illustrer ma conception du bon usage des psychiatres par les orthopédistes, non seulement les orthopédistes mais tous les somaticiens, je m'appuierai sur cette observation qu'un collègue a bien voulu me confier.

Il s'agit d'une jeune femme de 24 ans qui consulte un orthopédiste pour la première fois en décembre 1990 pour lombalgies et douleurs de la jambe droite. De manière très laconique, la première ligne de son dossier comporte, soulignée en rouge, la mention "personnalité névrotique". Cette expression est tirée directement de la lettre du généraliste qui l'envoie et qui précise bien que c'est "une personnalité névrotique en voie de décompensation". Il ne donne pas de détail ; on ne sait rien sur sa vie personnelle, on ne sait pas si elle est mariée, si elle travaille, si elle a des antécédents en-dehors d'une chute sur le dos un an et demi auparavant. L'observation s'intéresse par contre à son dos qui fera l'objet d'une intervention pour spondylolyse L5 deux mois plus tard. Curieusement, l'examen clinique avait montré que ses douleurs n'étaient pas centrées sur L5, mais plus haut. Néanmoins cette personnalité névrotique, qui pour l'instant n'a pas d'autre personnalité, sera opérée. Tout se passe bien, à ceci près que les plaintes douloureuses vont décupler, compliquées d'une infection urinaire et d'une réaction allergique imputée au nickel. A propos du nickel, permettez- moi d'ouvrir une parenthèse psychosomatique ; quand je faisais des consultations en dermatologie, j'ai examiné plusieurs femmes qui faisaient des allergies au nickel ou autres métaux rentrant dans la composition des bijoux fantaisies. Le remède était simple : il suffisait de leur prescrire des bijoux en or, je ferme la parenthèse. J'en reviens à ma malade qui se plaint de douleurs dans le genou gauche. On suspecte une chondropathie rotulienne et on décide de pratiquer une arthroscopie qui ne montre rien et qui est suivie de complication infectieuse sous forme d'arthrite à entérobacters, laquelle va nécessiter coup sur coup deux nouvelles interventions, dont la seconde au décours d'un séjour en réanimation pour choc septique. Il va de soi que ses plaintes ne vont pas décroître et l'observation mentionne, je cite à nouveau : "de toute évidence, énorme disproportion entre l'objectif et le subjectif, comportement névrotique, hypertrophie tout, exigences +++ , problème relationnel familial ?".

Dans ce contexte dramatique, vont entrer en scène deux nouveaux personnages : tout d'abord le mari "agressif et revendicateur". Je vous fais remarquer que c'est la première fois qu'il en est fait mention ; les comptes-rendus opératoires indiquaient auparavant alternativement mademoiselle ou madame. Intervient ensuite le psychiatre vacataire un peu comme un pompier venu éteindre l'incendie, ce qu'il s'efforce de faire en arrosant la patiente de Laroxyl et en prodiguant quelques bonnes paroles à chacun.

Aux chirurgiens, il conseille de ne pas se sentir coupable ou du moins de ne pas le laisser paraître ; au mari, il explique que la malade pourra bénéficier de son aide psychothérapique et le dissuade de porter plainte. En s'entretenant avec la patiente, il découvre selon ses propres termes "une jeane hystérique qui ne manque pas d'arguments pour séduire les chirurgiens mais qui se sent devenue victime impuissante et qui risque de virer à la paranoïa". Il découvre également qu'elle a deux jeunes enfants, Dieu seul sait qui s'en occupe pendant les longs mois d'hospi- talisation ? Le psychiatre apprend également qu'elle a développé une véritable toxicomanie aux antalgiques depuis l'âge de 22 ans, qu'elle prend de façon permanente à forte dose du Temgésic et bien d'autres choses encore. Mais le pot aux roses, si on peut dire, est découvert par les chirurgiens et par les infirmières qui constatent qu'elle n'a plus de température dès lors qu'on la surveille au moment où on la mesure. On a ainsi la preuve qu'elle a triché et on a aussi de bonnes raisons de penser qu'elle a provoqué elle-même son arthrite du genou.

De cette histoire, qui je le crains n'est pas terminée, je ferai de brefs commentaires : en premier l'intervention malheureusement tardive du psychiatre en octobre 1992, alors que dès décembre 1990 le dossier indiquait "personnalité névrotique". Ce retard est à interpréter selon moi non comme une négligence, mais par le fait qu'un entretien avec un psychiatre garde encore un caractère infamant principalement pour les malades. En discutant avec les uns et les autres ici, j' ai remarqué à quel point on évitait le mot psychiatre, pour lui préférer psychologue, psychosomaticien, ou que sais-je encore. Plusieurs traits cliniques constituent dans le cas que j'ai exposé des indices de troubles de la personnalité de type hystérique. Non seulement les plaintes algiques dont la disproportion est patente mais aussi la consommation médicale frénétique, les problèmes personnels qui dans un premier temps avaient étê occultés, l'hystérique redoutant par-dessus tout de ne pas être traité comme un vrai malade, mais d'être "recalé" à l'examen d'entrée au bloc opératoire.

Pour conclure, je voudrais vous faire un aveu. Mon intervention m'avait mis devant un choix difficile : soit je disais le fond de ma pensée et je prenais le risque de ne jamais vous revoir, soit je prenais le parti de vous caresser dans le sens du poil pour ménager vos susceptibilités. En fait, c'était un faux problème. La plupart d'entre vous, j'en suis convaincu, n'attendent des psychiatres aucun ménagement, de même que je n'en attends aucun de vous. 